\title{
THE SURGICAL TREATMENT OF EPILEPSY
}

\author{
JoHN ANDREw, M.B., F.R.C.S. \\ Regional Centre for Neurology and Neurosurgery, Oldchurch Hospital, Romford, Essex
}

THE surgical treatment of epilepsy is reserved for those cases in which the seizures are 'focal ' in origin, and are symptomatic of an underlying local pathological condition in the brain. Focal or generalized seizures may complicate overt expanding intracranial lesions, but they will not be considered in this paper. Suffice it to say that one-third of patients developing epilepsy in adult life will have intracranial neoplasms, and all such patients must be investigated bearing this possibility in mind.

The purpose of this paper is therefore to consider those cases of focal epilepsy not due to an obvious expanding lesion, and their investigation and management. The underlying pathological conditions to be considered are usually atrophic. These include meningo-cerebral scars, often resulting from old trauma or inflammatory states, birth injuries, so frequently sited in the temporal lobes, and vascular lesions.

The work of Hughlings Jackson forms the basis of the rational treatment of epilepsy by surgical means. The detailed investigations of Penfield have led to a better understanding of the function of the human cerebral cortex and has made the surgical treatment a practical proposition, so that it is carried out in all neurosurgical centres today.

For the purpose of this paper we may conveniently, if artificially, divide the cases for surgery into three groups: hemispheric lesions not involving the temporal lobe; temporal lobe or psychomotor epilepsy; and epilepsy associated with infantile hemiplegia.

\section{Hemispheric Lesions other than in the Temporal Lobe}

Experimental data obtained from animals as to cortical localization form the basis of human observations and the treatment of epilepsy. Observations during the excision of cortical or meningo-cerebral scars in the human have led to a more precise knowledge of cortical representation. Most of this has been derived from the work of Penfield and his colleagues, and will be referred to freely.

Again, cases of obvious expanding lesions will not be included. The pathological processes giving rise to focal epilepsy are birth injury, trauma, vascular disease and its sequelæ, and cieatrix following inflammation. Porencephalic cysts following old head injuries, or vascular accidents, may be found associated with an epileptogenic focus. Arterio-venous malformations are rarely encountered. A simple knowledge of cortical representation will suggest. the site of origin of most focal or symptomatic seizures. A generalized convulsion may be caused by a focus almost anywhere in the hemispheres, but may be preceded by a classical Jacksonian 'March', either motor, sensory or sensory-motor. These 'Marches' may remain unilateral, and are not necessarily followed by unconsciousness. An autonomic aura may arise from wide areas of the cortex, and speech disturbances likewise in the dominant hemisphere.

Investigation includes careful history-taking and neurological examination, electro-encephalogram recordings and plain skull $\mathrm{X}$-rays and air studies; angiography is called for when a vascular malformation is suspected.

The most careful assessment is called for in those patients, whose epilepsy is not controlled by medication. The excision of an extensive lesion from the Rolandic or speech area may cure the fits but leave a grossly disabled patient.

There have been no recent advances in the surgical technique in the removal of hemispheric scars. If possible the operation is carried out under local anæsthesia. A generous bone flap is cut to expose the lesion and surrounding brain. Electrocorticographs are made and the area of abnormality is outlined. Stimulations are then carried out. These may give two pieces of useful information. Firstly, the focus for the epilepsy may be found. Secondly, the sensory and motor cortex can be delineated if close to the lesion, and outlining these may prevent their inevitable damage and severe neurological deficit.

It is from the information gained from cortical stimulation and excisions that advances in the knowledge of cerebral localization and function have been made. 
The most important findings may thus be summarized from Penfield and Jasper (1954).

I. Precentral gyrus: Although this has to do with contralateral movements, its stimulation may evoke sensation on the opposite side of the body. Its removal does not necessarily result in hemiplegia, but renders complicated or skilful movements of the contralateral limbs impossible.

2. Postcentral gyrus: This is essential for the discriminatory elements in contralateral sensation. Stereognosis is one of the most important of these. Its stimulation may also give rise to contralateral motor activity. Its removal interferes with perception of fine sensation in the limbs, maximal peripherally.

It is believed that the postcentral gyrus is an indispensable relay station of afferents to a centrencephalic or integrating system; the precentral gyrus is thought to be indispensable for complicated movements and subserves a higher centre in the upper brainstem.

3. $A$ second sensory area has been found on the superior bank of the Sylvian fissure, in which there is ipsilateral as well as contralateral representation. It is thought to be related to voluntary movement. Its excision results in no detectable deficit.

4. A supplementary motor area has been demonstrated on the medial aspect of the hemisphere above the cingulate gyrus and in front of the Rolandic zone. Its stimulation causes postural or rhythmic movements of either side of the body; vocalization; inhibition of voluntary action or of speech; general body sensations; pupillary alteration.

Speech Areas. Four cortical areas have been demonstrated: one in the low post-frontal zone (Broca), one behind the sensory strip in the parietal lobe, one in the posterior temporal region and one in the superior frontal area in front of the motor foot area.

Visual Function. Stimulation of the occipital lobes produces light flashes, or geometrical patterns, as does a seizure originating here. This type of hallucination may be distinguished from that resulting from a temporal lobe seizure or stimulation when a familiar scene or object may be perceived or relived.

Autonomic responses are obtained from stimulation along the Rolandic strip, insula and cingulum. Auditory, uncinate, vestibular and memory function will be considered with the section on temporal lobe epilepsy.

\section{Temporal Lobe Epilepsy}

This form of epilepsy has stimulated the interest of neurologists, neurosurgeons, pathologists -and psychiatrists and neurophysiologists. Since the days of Hughlings Jackson, when 'drear̄y states' were thought to be of temporal origin, numerous strides have been made.

The pattern of temporal lobe seizures, or psychomotor epilepsy, varies greatly. The focus of abnoimality may determine the type of attack. Whether or not there is a generalized convulsion the primary manifestation of a seizure is psychic This may be followed by a period of altered consciousness or loss of consciousness. The psychic phenomena include illusions, hallucinations, emotional disturbances or forced thinking (Penfield and Jasper, 1954). The illusions include visual, auditory or labyrinthine distur bances, déjà vu phenomena and feelings of moteness. The hallucinations may be visual, auditory or uncinate, and previous experiences may be relived.

Many sufferers from psychomotor epilepsy show behaviour disturbances which are sometimes so severe that institutional treatment becoms necessary. Others may even commit criminal actions during periods of automatism following the seizures.

The investigation of these cases after caren history-taking and neurological examination is follows:

1. Plain $X$-rays of skull. Calcification ma be seen in one temporal lobe. This may occur an old inflammatory lesion, a hamartoma or ofther tumours. The middle cranial fossa may be sm곙 on one side in cases of incisural sclerosis (Earde, Baldwin and Penfield, 1953).

2. Pneumo-encephalography. Every attempt $\stackrel{0}{\exists \mathrm{s}}$ made to fill both temporal horns to compare the्fir size and situation. Thus there may be dilatation of one horn as a result of atrophy, or displacem by a neoplasm.

3. Carotid angiography. This investigation ${ }_{3}$ is not indispensable, but is indicated if the presenced a neoplasm or an angiomatous malformation $\mathrm{Fis}_{\mathrm{s}}$ suspected.

4. Electro-encephalography. This is, of course, the most important of the special investigations. In addition to the routine scalp recordings, sphenoidal electrodes are inserted under loel anæsthesia through the cheek, so that the tip of the needle lies on the sphenoid to record activity from the undersurface of the anterior end of the tengporal lobe. Recordings are made at rest, undeger the influence of barbiturate-induced sleep, aod occasionally during stimulation with the strowscope or even such drugs as Metrazol. The slefp records are of great value. Spike or delta activiny may be accentuated or facilitated immediateby after the period when the effect of the shoatacting barbiturate is wearing off. A diseased af a of cortex may fail to produce the fast activigy 
induced by the drug in all other areas of the cerebral hemisphere.

In more obscure cases, where routine electroencephalograms are difficult to interpret, it is possible to place electrodes under the temporal lobe, and to insert depth electrodes, through a trephine opening, bringing the leads out through the scalp for serial recordings over a period of weeks.

Those cases considered suitable for surgery, that is temporal lobectomy, will all have been given a long trial of medical treatment first, and their attacks not brought under control. Serial electroencephalograms will have been made.

The factors favouring surgery include reduction of barbiturate-fast rhythm beneath one temporal lobe, or a unilateral sphenoidal spike focus on electroencephalography; a diseased temporal lobe in the non-dominant hemisphere; and fits occurring in a patient with a normal or aggressive personality. The contraindications include a spike focus, not at the sphenoidal or mastoid electrode, in the dominant side, multiple foci of epileptic activity without dominance at one site; cortical atrophy; a hysterical or inadequate personality (Hill, 1958).

Bilateral sphenoidal abnormality, on repeated examination without a clear preponderance is a contraindication. Bilateral temporal ablation may lead to severe intellectual or personality change. The classical changes in monkeys (Kluver and Bucy, 1939) following this procedure include a complete inability to memorize or to learn from experience and grossly depraved habits.

The operation of temporal lobectomy may be carried out under local or general anæsthesia. The pioneer work of Penfield was carried out with the patient awake. Cortical stimulation generally reproduced the seizure pattern, and in this way much was learnt of functional localization within the temporal lobe. A sufficiently large scalp and bone flap is cut to expose the whole temporal lobe down to the floor of the middle cranial fossa, and the adjacent areas of the frontal and parietal lobes. Inspection of the brain reveals any surface $a b-$ normality. Electrodes are then placed over the surface of the brain and under the temporal lobe at suitable sites, and depth electrodes may be inserted into the amygdala or uncus. If the patient is anæsthetized, he is kept as light as possible; 2 or 3 cc.s of a $5 \%$ pentothal solution may be given. This will at first flatten the electrical activity, and then fast rhythms will appear, which may be absent over the abnormal area. As recovery occurs spike activity may then be seen from this area. The leads from which the greatest spike amplitude is seen denote the diseased area, as will a phase-reversal.

When the electrocorticograms have confirmed that the maximal abnormality is in the anterior temporal region, a lobectomy is carried out. The pia-arachnoid and cortex are incised just below the outer end of the Sylvian fissure along the upper bank of the superior temporal convolution; this incision is carried in a coronal direction across the lobe between 5.5 and $6.5 \mathrm{~cm}$. behind the tip of the lobe. The ventricle is cut across. Dissection under the insula enables the lobe to be removed complete with the uncus and anterior end of the hippocampus.

The results of surgery have been fairly gratifying. Northfield (1958) reports a good or fair improvement in epilepsy in over $60 \%$ of cases during a follow-up of several years. The mental symptoms have been benefited in over half the cases.

The complications include a small but variable upper quadrantic homonymous field defect in the opposite field of vision; when the dominant side has been operated on there is some mild nominal dysphasia and a deficit of learning by auditory modalities (Falconer, 1958).

\section{Case Report}

C.H., a boy of eleven, was born by normal delivery and had no family history of epilepsy. He developed normally, although he was somewhat backward until the age of nine when he commenced to have epilepsy. His seizures were of two types. The first was ushered in by an unpleasant taste, and he would then feel remote, and although vaguely aware of his environment was unable to speak or carry out any action. These attacks would last for two to three minutes, and would occur up to six times daily. The second type of attack was a typical grand mal seizure, occurring up to six times nightly. Since the onset of the attacks the boy's personality had completely altered. He became vicious, began to thieve and would fail to attend school. He lied and had numerous temper tantrums. Repeated electro-encephalograms over six months confirmed a persisting left, dominant, spike focus at the sphenoidal lead. All the routine anticonvulsant drugs failed to control his fits; his behaviour deteriorated. At operation a normal-appearing left temporal lobe was seen, and a $5.5-\mathrm{cm}$.-long lobectomy was performed. Histologically it showed sclerosis in the uncus. One year later there had been no recurrence of either type of attack. The boy's character returned to that before the onset of his illness, and the only operative complications were a slight nominal dysphasia and impairment of auditory learning. In spite of this he has returned to his former position at school.

\section{Pathological Changes in the Temporal Lobes}

The most common abnormality is found on serial histological examination, when sclerosis or gliosis is seen in the uncus, amygdala or Ammon's horn. There is an increase of glia and an overall loss of neurones. The condition is referred to as incisural sclerosis. Earle et al. (1953), reported this finding in $63 \%$ of 157 cases operated on. They suggest birth trauma as the ætiological factor. Excessive moulding of the fotal head causes herniation of the uncus through the incisural 
opening at the tentorium. Branches of the posterior cerebral, anterior choroidal and middle cerebral arteries crossing the free edge of the tent are so placed that herniation at birth would compress them against the free edge and result in ischæmic changes in the temporal lobe.

Uncal herniations occurring from acute supratentorial expanding lesions may also result in psycho-motor epilepsy commencing many years after the acute illness. The present writer is reporting two such cases. One was a boy who developed classical temporal seizures ten years after he had been seriously ill with a frontal lobe abscess, and the other was a man whose attacks -commenced six years after acute ipsilateral cerebral compression following a head injury.

The abnormalities seen within the excised temporal lobe are small unsuspected gliomata, hamartomata, and angiomata.

Stereotaxic investigation and treatment is still in the experimental stage and only carried out at a few centres, under radiological control. Electrodes may be placed into the amygdala, uncus, hippo- campus, and first temporal convolution. These may be left in situ and brought out through the scalp for serial recordings and even stimulation If a small focus of marginal abnormality is foun $q^{\mathbb{Q}}$ this may be destroyed electrolytically, thus avoid ing open craniotomy and full temporal lobectomys

\section{Epilepsy Associated with Infantile Hemiplegia}

Krynauw (1950) reported 12 cases of infantile hemiplegia in which the diseased contralaterad hemisphere was excised. The majority of these suffered from epilepsy in one of its forms, and in all the cases operated on, the epilepsy ceased with $\overrightarrow{\vec{\omega}}$ out medication. There was improved poste operative motor power with lessened spasticitis and clumsiness and a marked improvement in. personality and behaviour. The experience ofy other neurosurgeons has not always been so en couraging. In these cases there has been a swing to a less radical procedure whereby only grossly atrophic and electrically abnormal areas of cortex are excised.

\section{REFERENCES}

Earle, K. M., Baldwin, M., and Penfield, W. (1953): Incisural Sclerosis and Temporal Lobe Seizures produced $\not \overrightarrow{0} \overline{0}$ Hippocampal Herniation at Birth, Arch. Neurol. Psychiat. (Chicago), 69, 27.

Falconer, M. (1958): Temporal Lobe Epilepsy, Proc. roy. Soc. Med., 51, 6r 3 .

Hrll, D. (1958): Temporal Lobe Epilepsy, Ibid., 51, 6 ro.

Kluver, H., and Bucy, P. C. (1939): Primary Analysis of Functions of the Temporal Lobe of Monkeys, Arch. Neurol Psychiat. (Chicago), 42, 979.

KRYNAUW, R. A. (1950): Infantile Hemiplegia Treated by removing one Cerebral Hemisphere, $\mathcal{F}$. Neurol. Neurosurgo Psychiat., 13, 243.

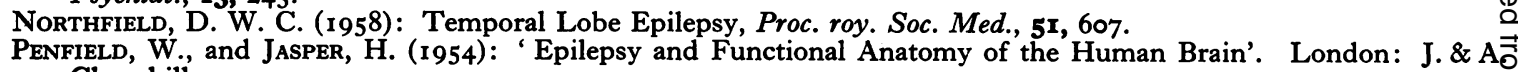
Churchill. 